\title{
TRANSFORMATION CONCEPTS FOR NATIONAL SECURITY IN THE 21st CENTURY
}

Williamson Murray

Editor

September 2002 